PHYSICAL REVIEW D 93, 109904(E) (2016)

\title{
Erratum: Earth-moon Lagrangian points as a test bed for general relativity and effective field theories of gravity [Phys. Rev. D 92, 064045 (2015)]
}

Emmanuele Battista, Simone Dell'Agnello, Giampiero Esposito, Luciano Di Fiore, Jules Simo, and Aniello Grado (Received 6 May 2016; published 31 May 2016)

DOI: 10.1103/PhysRevD.93.109904

Equations (4.62)-(4.64) should read as

$$
\begin{aligned}
& r_{1}=3.263762881738878 \times 10^{8} \mathrm{~m}, \\
& r_{2}=4.489205600344675 \times 10^{8} \mathrm{~m}, \\
& r_{3}=3.816747156939623 \times 10^{8} \mathrm{~m} .
\end{aligned}
$$

General relativity corrections on Newtonian Lagrangian points, reported in Eqs. (4.65)-(4.67) should read as

$$
\begin{gathered}
R_{1}^{(\mathrm{GR})}-r_{1}^{(N)}=R_{1}-r_{1}=0.19 \mathrm{~mm} \text { at } L_{1}, \\
R_{2}^{(\mathrm{GR})}-r_{2}^{(N)}=R_{2}-r_{2}=-0.32 \mathrm{~mm} \text { at } L_{2}, \\
R_{3}^{(\mathrm{GR})}-r_{3}^{(N)}=R_{3}-r_{3}=-0.04 \mathrm{~mm} \text { at } L_{3} .
\end{gathered}
$$

The first column of Table I should read as

TABLE I. Distances $r_{i}$ from the Earth and planar coordinates $\left(\xi_{i}, \eta_{i}\right)$ of the planetoid at all Lagrangian points $L_{i}$ in the classical Newtonian theory, general relativity, and the quantum regime, the latter being obtained through the Lagrangian $L_{V}(5.11)$ and with the coefficients of the bound-states potential $\kappa_{1}=-1 / 2$ and $\kappa_{2}=41 /(10 \pi)$ taken from Ref. [12].

\begin{tabular}{lc}
\hline \hline & Newtonian gravity \\
\hline$L_{i}$ & Details \\
& $r_{1}=3.263762881738878 \times 10^{8} \mathrm{~m}$ \\
$L_{1}$ & $\xi_{1}=3.217044369761366 \times 10^{8} \mathrm{~m}$ \\
& $\eta_{1}=0 \mathrm{~m}$ \\
$L_{2}$ & $r_{2}=4.489205600344675 \times 10^{8} \mathrm{~m}$ \\
& $\xi_{2}=4.442487088367163 \times 10^{8} \mathrm{~m}$ \\
& $\eta_{2}=0 \mathrm{~m}$ \\
$L_{3}$ & $r_{3}=3.816747156939623 \times 10^{8} \mathrm{~m}$ \\
& $\xi_{3}=-3.863465668917136 \times 10^{8} \mathrm{~m}$ \\
$L_{4}$ & $\eta_{3}=0 \mathrm{~m}$ \\
& $r_{4}=3.844000000000000 \times 10^{8} \mathrm{~m}$ \\
& $\xi_{4}=1.875281488022488 \times 10^{8} \mathrm{~m}$ \\
$L_{5}$ & $\eta_{4}=3.329001652147382 \times 10^{8} \mathrm{~m}$ \\
& $r_{5}=3.844000000000000 \times 10^{8} \mathrm{~m}$ \\
$\xi_{5}=1.875281488022488 \times 10^{8} \mathrm{~m}$ \\
$\eta_{5}=-3.329001652147382 \times 10^{8} \mathrm{~m}$ \\
\hline
\end{tabular}


whereas Table II becomes

TABLE II. Corrections to the distances of $L_{1}, L_{2}$, and $L_{3}$ from the Earth and to the planar coordinates of $L_{4}$ and $L_{5}$. In the first column we have the general relativity corrections to Newtonian theory as obtained from Eqs. (4.38), (4.50), (4.16), and (4.17), respectively. The second column shows quantum corrections to general relativity given by the Euler-Lagrange equations involving the Lagrangian $L_{V}(5.11)$ and the quantum coefficients of the bound-states potential. The last column displays quantum corrections to Newtonian values calculated as the algebraic sum of the corresponding quantities in the two previous columns.

\begin{tabular}{lccc}
\hline \hline$L_{i}$ & General relativity-Newton & Quantum-general relativity & Quantum-Newton \\
\hline$L_{1}$ & $0.19 \mathrm{~mm}$ & $-0.62 \mathrm{~mm}$ & $-0.43 \mathrm{~mm}$ \\
$L_{2}$ & $-0.32 \mathrm{~mm}$ & $-0.39 \mathrm{~mm}$ & $-0.71 \mathrm{~mm}$ \\
$L_{3}$ & $-0.04 \mathrm{~mm}$ & $-1.48 \mathrm{~mm}$ & $-1.52 \mathrm{~mm}$ \\
$L_{4}$ & $(2.73 \mathrm{~mm},-1.59 \mathrm{~mm})$ & $(-1.46 \mathrm{~mm},-0.86 \mathrm{~mm})$ & $(1.27 \mathrm{~mm},-2.45 \mathrm{~mm})$ \\
$L_{5}$ & $(2.73 \mathrm{~mm},-1.59 \mathrm{~mm})$ & $(-1.46 \mathrm{~mm},-0.86 \mathrm{~mm})$ & $(1.27 \mathrm{~mm},-2.45 \mathrm{~mm})$ \\
\hline \hline
\end{tabular}

In this table lines 4 and 5 coincide with those of our original paper. Thus, general relativity corrections to Newtonian values for $L_{1}, L_{2}$, and $L_{3}$ are actually very small. The last ten lines of the Abstract, beginning with the words In the latter case, for the point ... should be deleted. Remarks (iii) and (iv) in Sec. VI B and the last 18 lines of Sec. VI, beginning with the words: In fact, given the large value ... should be deleted. 